\title{
Characterization of temporal soft tissue space to aid the design of implants aimed at restoring function in facial paralysis
}

\author{
Shaheen Hasmat ${ }^{1,2,3,4}$, Don L. Wanasinghe ${ }^{1}$, Kai Cheng $^{3}$, Gregg J. Suaning ${ }^{5}$, Nigel H. Lovell ${ }^{6}$, Tsu-Hui \\ (Hubert) Low ${ }^{1,2,3,4}$, Jonathan R. Clark ${ }^{1,2,3,4}$ \\ 1Sydney Medical School, Faculty of Medicine and Health Sciences, The University of Sydney, Camperdown, NSW 2006, Australia. \\ ${ }^{2}$ The Sydney Head and Neck Cancer Institute, Department of Head and Neck Surgery, The Chris O'Brien Lifehouse, Camperdown, \\ NSW 2050, Australia. \\ ${ }^{3}$ Royal Prince Alfred Institute of Academic Surgery, Sydney Local Health District, Sydney, NSW 2050, Australia. \\ ${ }^{4}$ Sydney Facial Nerve Service, The Chris O'Brien Lifehouse, Camperdown, NSW 2050, Australia. \\ ${ }^{5}$ School of Biomedical Engineering, University of Sydney, Camperdown, NSW 2006, Australia. \\ ${ }^{6}$ Graduate School of Biomedical Engineering, University of New South Wales Sydney, NSW 2052, Australia.
}

Correspondence to: Dr. Shaheen Hasmat, The Chris O'Brien Lifehouse, 119-143 Missenden Road Camperdown, New South Wales 2050, Australia. E-mail: shas9044@uni.sydney.edu.au

How to cite this article: Hasmat S, Wanasinghe DL, Cheng K, Suaning GJ, Lovell NH, Low T, Clark JR. Characterization of temporal soft tissue space to aid the design of implants aimed at restoring function in facial paralysis. Plast Aesthet Res 2021;8:17. http://dx.doi.org/10.20517/2347-9264.2020.221

Received: 19 Dec 2020 First Decision: 27 Jan 2021 Revised: 30 Jan 2021 Accepted: 9 Feb 2021 Published: 18 Mar 2021

Academic Editor: Raúl González-García Copy Editor: Yue-Yue Zhang Production Editor: Yue-Yue Zhang

\begin{abstract}
Aim: Facial paralysis inflicts devastating functional and aesthetic deficits. Several solutions are being developed, including implantable bionics to correct paralytic lagophthalmos. The temporal fossa has been postulated to be a suitable location for such devices. Anatomical studies of this fossa have limited application in the design of implants with complex internal components that are constrained by specific functional requirements. In this study, we assess the variation in temporal fossa volumes that could be utilized by a functional implantable device.
\end{abstract}

Methods: CT scans of 18 hemifaces were used to create a 10-point template for measuring tissue thickness in the temporal fossa. Using this data, linear models were used to perform a volumetric analysis of the temporalis muscle and temporal fat pad concerning key anatomical landmarks.

Results: The estimated temporalis muscle, temporal fat, and total combined volumes were $19.2 \mathrm{~mL}(95 \% \mathrm{Cl}$ : 10.4 32.9), $10.3 \mathrm{~mL}(95 \% \mathrm{Cl}: 6.1-16.1)$, and $29.5 \mathrm{~mL}$ (95\% Cl: 16.7-48.9) respectively, consistent with other publications. The temporalis muscle volume increases rapidly and then plateaus moving posteriorly along the zygomatic arch 
and superiorly along the lateral orbital rim. Whereas the temporal fat increases similarly along the lateral orbital rim, it increases at a uniform rate along the zygomatic arch.

Conclusion: Simple geometric modelling of the functional soft tissue space in the temporal fossa is feasible and can be readily applied to aid in the development of implantable devices.

Keywords: Blink, facial palsy, prosthesis, reanimation, eyelid

\section{INTRODUCTION}

The last seven decades have seen the introduction of numerous implantable solutions for dynamic reanimation in facial nerve paralysis $(\mathrm{FNP})^{[1-3]}$. The head and neck is a region of high visibility with dense anatomy and complex geometry that makes the placement of a prosthesis challenging. Several authors have recognized the temporal region as a suitable space for this purpose. Tollefson and Senders proposed using electro-active polymers as a muscle substitute for restoring dynamic eye closure in $\mathrm{FNP}^{[2]}$. The device is positioned in the temporal fossa from where it moves the eyelids through the application of an eyelid sling, akin to temporalis muscle transfer. Hasmat et al. ${ }^{[3]}$ attempted restoring physiological blinking kinematics in cadaveric models using an implantable magnetic actuator confined to the temporal fossa. More recently, Jowett et al. ${ }^{[1]}$ described a hybrid approach whereby contralateral facial nerve signals are used to drive an innervated free muscle flap through use of nerve electrodes. Again, the processing unit for controlling the electrodes is placed in the temporal fossa. However, none of these methods has described the geometric constraints of this space and how this can be used.

The temporal soft tissue space is an intuitive choice of implant location for several reasons. One, the temporal fossa is largely occupied by the temporalis muscle and fat, which can be removed without significant functional consequences ${ }^{[4]}$. Two, the soft tissue is void of critical structures except for frontal branches of the facial nerve, which would be non-functional where the use of implantable solutions is indicated, or can be reflected inferiorly. Finally, the proximity to affected structures makes it ideal; for instance, actuators described to restore eye-blink by tensioning of an eyelid sling mechanically necessitates a lateral positioning of the actuator to enable eye closure ${ }^{[2,3]}$.

For cosmetic placement in the temporal region, the device must be confined to the limits of removable soft tissue with minimal external deformity. Anatomical studies to date have performed complex geometrical assessments of the total potential space in the temporal region for various applications. Morphometry of the temporalis muscle and temporal fat are described for facial approximation caseworks ${ }^{[5,6]}$. The temporalis muscle bulk is measured as an index of clinical health where its mass is reported as a predictor of perioperative morbidity as well as a marker of survival in oncology patient ${ }^{[7-9]}$. Finally, the temporalis muscle and adjacent fat layers are quantified for augmentation purposes ${ }^{[10-12]}$. Computer tomography (CT) and magnetic resonance imaging (MRI) are the most commonly used imaging modalities in settings where image processing and segmentation is applied for volumetric reconstruction of the temporalis and the adjacent fat ${ }^{[10,13]}$. These volumetric analyses, however, have limited application in implants with complex internal components that are constrained by specific functional requirements, especially those with moving parts. To aid the design of these implants, the complex biological space needs to be simplified and quantitatively characterised in relation to fixed structures, i.e., bones, for accurate placement and function of the device.

In this study, we assess the variation in temporal fossa volume that could be utilized by a functional implant designed to correct paralytic lagophthalmos in FNP. To enable the transfer of this information into the 
Table 1. Mean soft tissue thickness in the temporal region.

\begin{tabular}{lllll}
\hline Tissue & \multicolumn{2}{c}{$\begin{array}{c}\text { Points along } \\
\text { zygomatic arch }\end{array}$} & \multicolumn{2}{c}{ Points along the lateral orbital rim } \\
\hline \multirow{3}{*}{ Fat } & Jugale point & Whitnall's tubercle & Frontozygomatic suture \\
& Jugale point & $9.4(3.3)$ & $6.6(3.1)$ & $4.5(2.1)$ \\
& Mid-arch & $9.2(1.3)$ & $6.3(2.2)$ & $3.9(2.0)$ \\
Temporalis & End of arch & $7.5(0.9)$ & $5.5(1.5)$ & $3.7(1.4)$ \\
& Jugale point & $14.7(2.8)$ & $12.6(2.4)$ & $11.1(2.4)$ \\
& Mid-arch & $10.5(3.4)$ & $8.0(1.9)$ & $7.8(2.3)$ \\
& End of arch & $4.3(0.9)$ & $4.0(1.2)$ & $4.0(1.3)$ \\
& "a" & $9.20(2.31)$ & & \\
\hline
\end{tabular}

The end of the zygomatic arch was measured as the point along the arch before it begins to widen posteriorly. Mid-arch refers to the point equidistant between the Jugale point and end of the arch. "a" as indicated in Figure 1 measures the transverse length from Jugale point to end of the groove at the lateral orbital rim which is occupied by the temporalis muscle. SD: Standard deviation.

design process of an implantable device, simplified mathematical models are developed that can provide an estimation of soft tissue volume and predict its anatomical distribution in relation to the local skeletal framework.

\section{METHODS}

This study was carried out following institutional ethics approval (HREC LNR18/RPAH/153) and patient consent was obtained for all images.

\section{Anatomical boundaries}

Uniform estimations of the temporal fossa boundaries were used to determine the effective space available that can accommodate implants with mechanical systems that require accurate positioning for successful device function. For example, an excursion of $6.5 \mathrm{~mm}$ is needed for complete eye closure using an eyelid sling ${ }^{[14]}$. Device parameters must negotiate the changing boundaries within the tissue space to achieve its desired function. Temporal fossa volume remains constant in adulthood and across genders, whereas variation in tissue height or thickness occurs largely as a result of changes in the temporalis muscle and the overlying fat pads ${ }^{[6]}$. Thus, the bony framework was used as parameters in models designed to measure the tissue constraints. Anatomically, the temporalis fossa is bounded superiorly by the inferior linea temporalis, posteriorly by the squamosal suture, anteriorly by the lateral orbital rim, and inferiorly by the inferior aspect of the zygomatic $\operatorname{arch}^{[6]}$. However, most implantable devices are limited to the temple region that is skeletally confined by the lateral orbital rim and superior margins of the zygomatic arch. The two bony columns meet at the Jugale point that was used as a reference point in the present study with two additional points selected along each column of bone as summarized in Table 1 and illustrated in Figure 1.

\section{Selection criteria}

Patients were included from a cohort of subjects undergoing scanning for virtual surgical planning for jaw reconstruction. Cases were excluded if they had musculoskeletal deformities in the maxilla or the temporal region or tumour extension into these regions. Cases were selected to include a broad range of ages and equal gender distribution.

\section{Soft tissue modelling}

Patient CT images (Siemens Somatom, Erlangen, Germany) were acquired (1 mm slice thickness, matrix of 512x512, PV phase) using an industry-standard scanning protocol (https://www.materialise.com/system/ files/resources/DSEM-CMF-1214-0050-1_LR_v4.pdf) and then saved in DICOM (Digital Imaging and Communications in Medicine) format. Image segmentation, thresholding, and surface model generation was conducted using 3D Slicer (https://www.slicer.org/) on a x64-based MSI GT80S 6QE workstation 


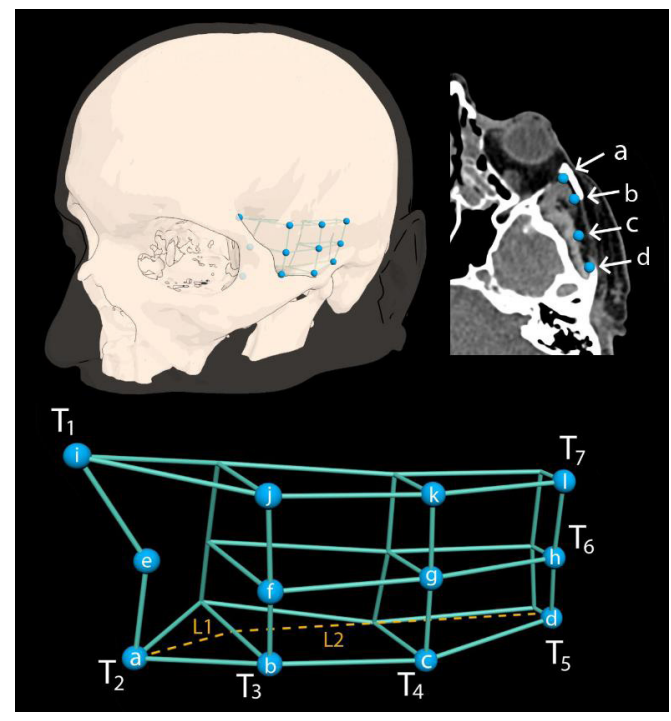

Figure 1. Modelling soft tissue volume in the temporal region. (A) Key anatomical landmarks are identified on the CT scan aided by 3D reconstruction of the scan. Tissue thicknesses are measured in reference to these points. (B) These are then laid out as coordinates across a template. The coordinates are used to generate linear equations that predict tissue thickness along different planes across the length $(\mathrm{T} 1,2,6,7)$ and height $(\mathrm{T} 3,4,5)$ of the soft tissue construct. These equations are as following. Temporal fat: $\mathrm{T} 1=0.55 / ; \mathrm{T} 2=1.44 \mathrm{l} ; \mathrm{T} 3$ $=-0.21 h+9.37 ; \mathrm{T} 4=-0.22 h+9.20 ; \mathrm{T} 5=-0.16 h+7.53 ; \mathrm{T} 6=-0.061 /+9.61 ; \mathrm{T} 7=-0.00861+4.18$. Temporalis muscle: $\mathrm{T} 1=0.028 l ; \mathrm{T} 2=$ $0.92 l ; \mathrm{T} 3=-0.15 h+14.6 ; \mathrm{T} 4=-0.12 h+10.2 ; \mathrm{T} 5=-0.008 h+4.2 ; \mathrm{T} 6=-0.35+14.9 ; \mathrm{T} 7=-0.261+11.08 . " h "$ is height along orbital rim and " $h$ " is length across the zygomatic arch in reference to the Jugale point. The latter divides the model into two lengths, L1 anteriorly and L2 posteriorly.

(NVIDIA GeForce GTX 980M, Processor Intel(R) Core(TM) i7-6920HQ CPU @ 2.90GHz, 2901 Mhz, 4 Core(s), 8 Logical Processor(s), Physical Memory and 36.7 GB Total Virtual Memory). The surface model was saved in OBJ file format and imported into Autodesk 2020 (Autodesk, Inc., San Rafael, CA). Surface model optimization was implemented to remove mesh parts irrelevant to the study.

Temporalis muscle and temporal fat thicknesses were measured across the selected coordinates as described above [Figure 1]. The two tissues were identified and delineated radiologically by differences in the Hounsfield scoring at the tissue interface. Muscle thickness at each point was measured as the length from the underlying bone surface to the muscle-fat interface, whilst fat thickness was calculated from the muscle-fat interface to the outer margins of the tissue. Tissue measurement at the coordinates was then used to generate linear models that predict tissue thickness in relation to the zygomatic arch and lateral orbital wall as demonstrated in Figure 1. Since the temporalis muscle and the temporal fat are not strictly confined to the limits of the above coordinate system, the linear models were extrapolated to estimate tissue thickness beyond these boundaries [Figure 2]. The volume segments reconstructed between the coordinates assume the shapes of triangular prisms or truncated pyramids, where the vertex is cut away at a plane parallel to the base. The models were imposed onto these simplified volumetric representations of the temporalis muscle and fat in relation to two axes, the zygomatic arch and lateral orbital wall, for clinical relevance. To achieve this, the volumetric models were determined as a function of a single axis, i.e., " $h$ " for height along the orbital wall and "l" for length along the zygomatic arch.

Formulae for volume estimation along the zygomatic arch are as following.

Temporalis muscle:

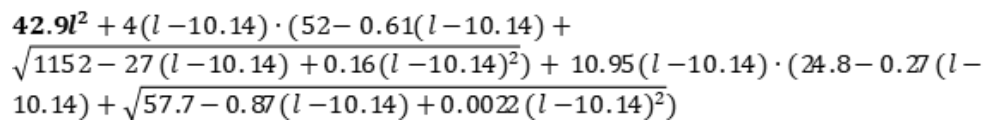




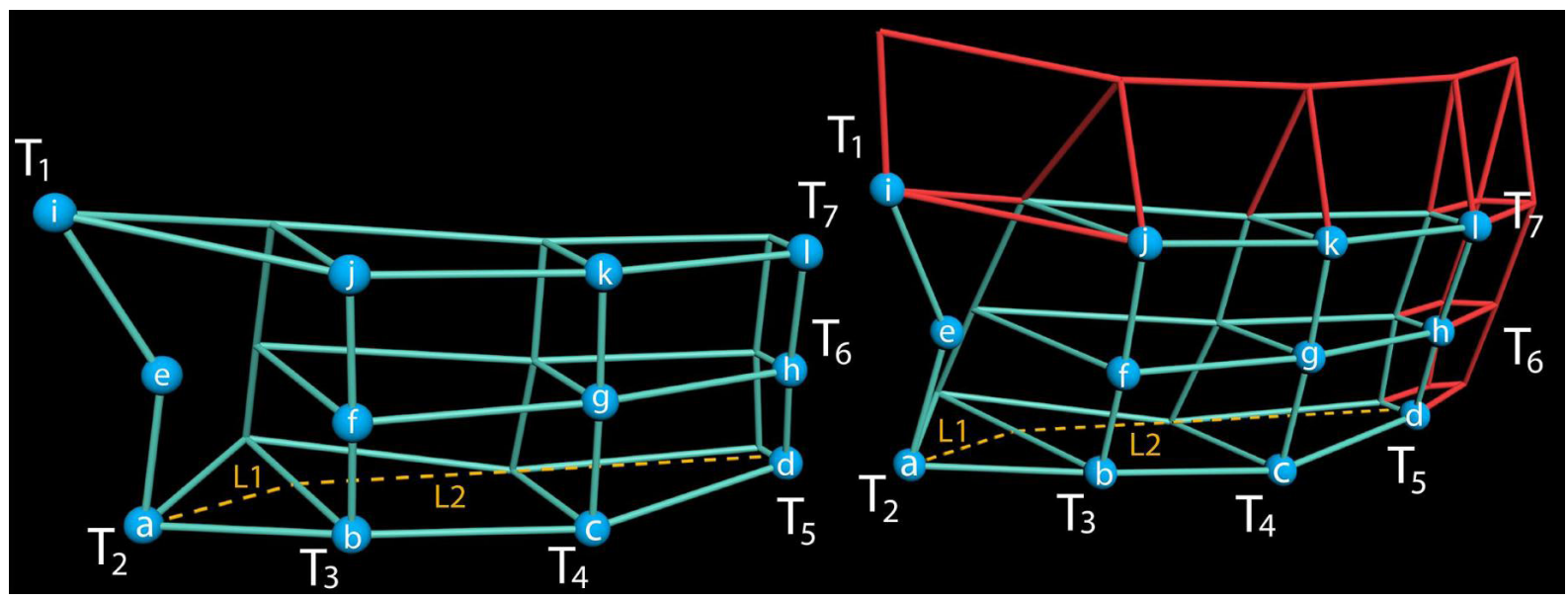

Figure 2. Linear models are extrapolated (red) to predict tissue thickness beyond the measured boundaries

Where $l \leq 10.14$ for the bold-lettered component of the model and $l>10.14$ for the remainder of the model. Temporal fat: $4 l \cdot\left(28-0.07 l+\sqrt{161-0.675 l+0.00053 l^{2}}\right)+3.22 l \cdot(836-0.0254 l+$
$\left.\sqrt{0.288 l-0.00087 l^{2}}\right)$

Formulae for volume estimation along the lateral orbital rim are as following.

Temporalis muscle:

$2.5 h \cdot\left(50-0.263 h+\sqrt{596-6.4 h+0.0171 h^{2}}\right)+2.5 h \cdot(29-0.124 h+$ $\left.\sqrt{171-1.14 h+0.00093 h^{2}}\right)+2.1 h \cdot(866-0.0124 h+$ $\left.\sqrt{3.22-0.039 h+0.000035 h^{2}}\right)+\left(\frac{h}{6}\right)(296-1.5 h+\sqrt{21904-222 h})$

Temporal fat:

$2.5 h \cdot\left(37.1-0.435 h+\sqrt{345-8.1 h+0.047 h^{2}}\right)+2.5 h \cdot(33.5-0.39 h+$ $\left.\sqrt{278-6.38 h+0.037 h^{2}}\right)+5 h \cdot\left(27-0.29 h+\sqrt{180-3.86 h+0.02 h^{2}}\right)$

\section{RESULTS}

A total of nine patients (five males and four females) with a median age of 71 years (range 39-84) were used to create 18 hemifaces for analysis [Figure 1]. The mean length of the zygomatic arch as measured from $b$ - $d$ was $29.3 \mathrm{~mm}$ (SD 2.1). The height of Whitnall's tubercle and frontozygomatic suture in relation to the Jugale point was $12.5 \mathrm{~mm}$ (SD 2.1) and $23.7 \mathrm{~mm}$ (SD 1.8), respectively. Temporalis muscle and temporal fat thickness measurements are listed in Table 1.

\section{Total volume}

The combined total volume of the temporalis muscle and fat was estimated to peak at $29.5 \mathrm{~mL}$ (95\% CI: 16.7-48.9). Figure 3 shows the cumulative tissue volume along the lateral orbital wall and the zygomatic arch. The total volume rises rapidly along both axes until arriving at an equal value of $14 \mathrm{~mL}$ at a distance of $19.5 \mathrm{~mm}$ from the Jugale point on either axis. The volume plateaus over a shorter distance along the zygomatic arch $(60 \mathrm{~mm})$ compared to the lateral orbital rim $(90 \mathrm{~mm})$.

\section{Temporalis muscle volume}

The maximum temporalis muscle volume as predicted by the models was $19.2 \mathrm{~mL}$ (95\%CI: 10.4-32.9). Muscle thickness is maximal near the junction of the lateral orbital rim and zygomatic arch, and declines 

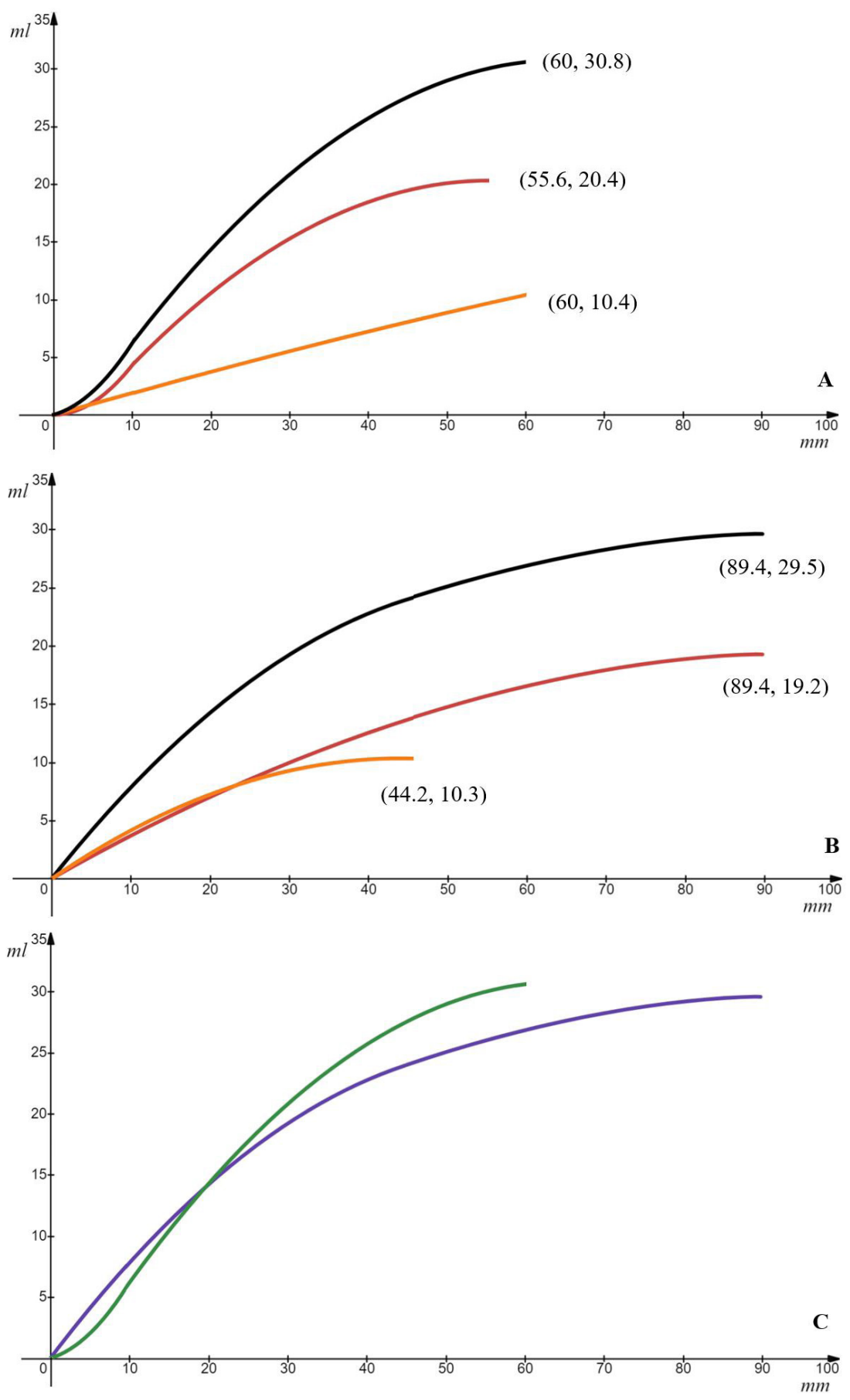

Figure 3. Cumulative soft tissue volume in relation to zygomatic arch and lateral orbital rim (red $=$ temporalis muscle, orange $=$ fat, black = combined total). (A) The maximum temporalis muscle volume predicted is $20.4 \mathrm{~mL}$ at a length of $55.6 \mathrm{~mm}$ along the zygomatic arch and the maximum fat volume predicted is $10.4 \mathrm{~mL}$ at a length of $60 \mathrm{~mm}$ with a combined total volume of $30.8 \mathrm{~mL}$. (B) The maximum temporalis muscle volume predicted is $19.2 \mathrm{~mL}$ at a length of $89.4 \mathrm{~mm}$ and the maximum fat volume predicted is $10.3 \mathrm{~mL}$ at a length of $44.2 \mathrm{~mm}$ along the orbital rim with a combined total volume of $29.5 \mathrm{~mL}$. (C) Total combined volume along the zygomatic arch axis (green) and the lateral orbital wall axis (purple). The discrepancy in volumes along the zygomatic arch and orbital axes occurs due to variation in individual tissue perimeters.

moving away from it. Volume calculations indicate that the temporalis rapidly gains volume moving along the axis of each bone before plateauing at $50 \mathrm{~mm}$ and $80 \mathrm{~mm}$ from the Jugale point along the zygomatic arch and lateral orbital wall, respectively, as shown in Figure 4. 


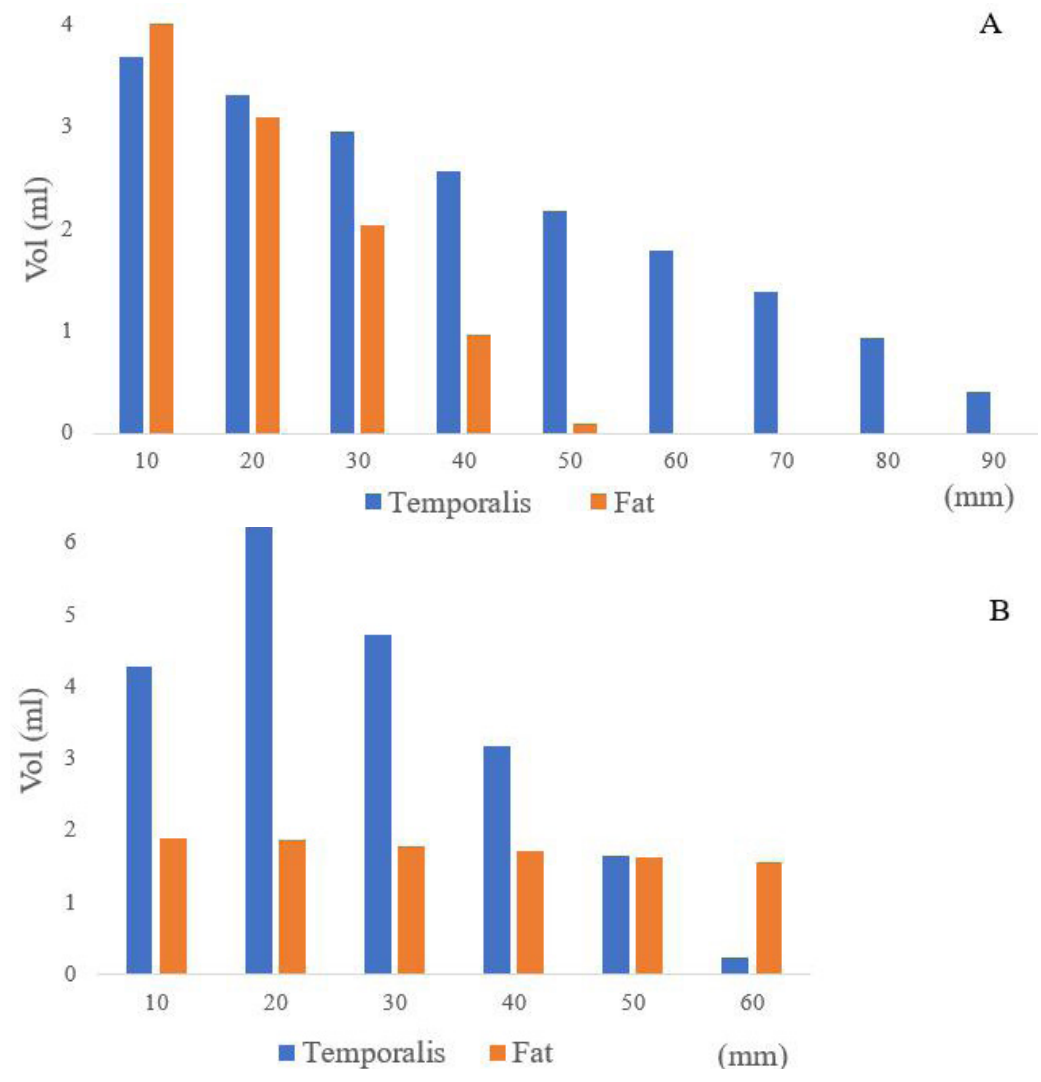

Figure 4. (A) Volume changes at $10 \mathrm{~mm}$ increments in reference to the Jugale point along the lateral orbital rim. The temporalis muscle and temporal fat volumes progressively increase moving closer to the Jugale point. (B) Volume changes at $10 \mathrm{~mm}$ increments in reference to the Jugale point along the zygomatic arch. The temporalis muscle progressively increases moving closer to the Jugale point, however, the fat volume changes at an equal rate.

\section{Temporal fat volume}

The estimated temporal fat volume was $10.3 \mathrm{~mL}$ (95\%CI: 6.1-16.1). The maximal thickness occurs near the junction of the lateral orbital rim and zygomatic arch, and declines moving posteriorly. The fat volume increases similarly as the temporalis muscle along the orbital rim, but more uniformly along the zygomatic arch (anterior to posterior) by $0.18 \mathrm{~mL} / \mathrm{mm}$. At $30 \mathrm{~mm}$ from the Jugale point along the orbital wall, the temporalis has achieved $51.8 \%$ of its total volume while the gain in temporal fat volume is $89.7 \%$ at the same height. Conversely, at $30 \mathrm{~mm}$ from the Jugale point along the zygomatic arch, the temporalis has achieved $75.0 \%$ of its total volume compared to a $53 \%$ gain in temporal fat volume at the same length.

\section{DISCUSSION}

This study demonstrates the feasibility of basic quantitative soft tissue modelling in the temporal fossa and its parametric relationship to the bony anatomy. The temporalis muscle increases rapidly in volume and then plateaus moving from anterior to posterior along the zygomatic arch and from inferior to superior along the lateral orbital rim. In contrast, whilst the temporal fat increases in the same manner along the orbital rim, its volume increases uniformly along the zygomatic arch at a rate of $0.18 \mathrm{~mL} / \mathrm{mm}$. The physiological distribution of muscle and fat in the temporal fossa has implications for designing implantable bionics that may be placed in the temporal region. We have avoided automatic image segmentation that applies intricate and irregular anatomical boundaries that are mechanically impractical and serve no functional use.

Kim and Matic ${ }^{[15]}$ identified the superficial fat pad as a quadrangular fan-shaped structure with a maximum height of $41 \mathrm{~mm}$ across 8 human cadaveric heads. Our model's prediction is in close range with 
a maximum height estimation of $45.7 \mathrm{~mm}$ in the living human. The greatest discrepancies occur at the peripheries of tissue where the temporalis muscle and temporal fat pads follow irregular boundaries. Given that peripheral tissue forms only a small proportion of total tissue mass, the discrepancies are unlikely to be relevant to implantable devices. Figure $3 \mathrm{C}$ demonstrates the effect of these discrepancies leading to small differences in the calculated total volume along the axis of the zygomatic arch and orbital rim.

The measured volume of temporalis muscle varies in the literature depending on the method used. The temporalis muscle and portions of the temporalis fat extend inferior to the zygomatic arch, beyond the calculation boundaries used in this study. Zhao et al. ${ }^{[6]}$ applied a modelling compound to fill the entire temporal fossa in 98 human cadaveric skulls and recorded a mean volume of $47.4 \mathrm{~mL}$. The authors also noted that the total volume of the temporal fossa remained constant across age. Volk et al ${ }^{[13]}$ performed facial muscle volumetry in healthy subjects using MRI and recorded a mean temporalis volume of $21 \mathrm{~mL}$ compared to our mean estimate of $19.2 \mathrm{~mL}$. The target muscle volume was manually measured by counting voxel numbers in all layers of the selected MRI orientation until the muscle was completely encircled. MRI is an excellent modality for soft tissue segmentation; however, CT more accurately identifies bony landmarks which has greater utility for implantable devices. Rinkinen et al. ${ }^{[7]}$ reported a temporalis fat pad volume of only $1.3 \mathrm{~mL}$ using CT images based on three anatomical landmarks. This is considerably less than the volume of $10.3 \mathrm{~mL}$ calculated in this study and the authors note that the value would vary if different landmarks were chosen. Several other reports limit soft tissue measurements in the region to only tissue thickness or diameter in a single plane ${ }^{[9,16]}$. Superimposing MRI soft tissue segmentation onto CT skeletal reconstruction might provide a more realistic and consistent volume estimation.

Volume estimations can also be made retrospectively as has been done in the reconstructive literature examining filler injections for temporal hollowing augmentation. Temporal depression is a well-known complication of temporalis muscle flaps. Cervelli et al ${ }^{[12]}$ used 3D photogrammetry to estimate the required fat graft volume in 45 patients with a temporal contour deformity. Using an over-corrected volume to account for fat absorption, an average of $30.8 \mathrm{~mL}$ was injected into each temple. This approximates our calculated mean total temporal soft tissue volume of $29.5 \mathrm{~mL}$. Idiopathic temporal hollowing is also described in healthy young adults. Huang et al. ${ }^{[11]}$ injected autologous fat graft into the temple of 96 young adults (mean age 34.4 years) with temporal hollowing attributed to fat atrophy. They reported a mean augmentation volume of $11.7 \mathrm{~mL}$, similar to our mean estimate of $10.3 \mathrm{~mL}$.

Although the present study measures fat in the temporal region as a single volume, soft tissue in the temporal fossa is partitioned across multiple distinct fascial planes ${ }^{[15]}$. Uniform nomenclature for the tissue planes is lacking in the literature, however, at least two separate fat pads are described. The deep temporal fat is continuous with the buccal fat and overlies the temporalis muscle. A more distinct superficial fat pad lies just deep to the superficial temporal fascia. A fibrous network arising from the superficial temporal fascia suspends the superficial fat unit whilst dividing it into lobules. A consistent vascular supply of the fat pad is reported through the deep and superficial fascial perforators from deep temporal and middle temporal arteries, respectively ${ }^{[15]}$. Kim and Matic ${ }^{[15]}$ identified the highest density of perforators occurring near the lateral orbital rim, zygomatic arch, and their junction across eight fresh-frozen cadaveric heads. When creating space for an implant, tissue removal may occur along the fascial planes until the target volume is achieved. The vasculature should be preserved to avoid tissue atrophy unless the given tissue is planned for replacement with the implant.

\section{Limitations}

Similar to companion studies, the calculations of the present study are limited by its sample size, imaging protocol, and observer biases. This study, however, describes a volumetric calculation using certain parameters. The calculation is purely a formula and hence it will not change with sample size. However, 
with increasing data points, the volume estimates will become more representative of the population and confidence intervals will decrease. The discrepancies resulting from different imaging protocols, and intrarater and inter-rater variability are evident in the literature. Using manual MRI segmentation, Goto et al. ${ }^{[17]}$ estimated a masseter volume of about $30 \mathrm{~mL}$ compared to $20 \mathrm{~mL}$ by Volk et al ${ }^{[13]}$, i.e., a $50 \%$ increase. Automation of this process has not achieved accuracy beyond $80 \%$ for temporalis muscle volumetry according to the literature ${ }^{[18]}$. The use of oncology patients may also introduce potential bias. Whilst the temporal fossa volume is reported to remain stable across gender and age ${ }^{[6]}$, it has been repeatedly documented that the muscle volume diminishes with cancer burden ${ }^{[7]}$.

\section{CONCLUSION}

A range of implantable bionics has been investigated in recent years for dynamic reanimation in FNP, most of which are proposed to sit in the temporal fossa. Although soft tissue volume in the temporal fossa is well described in the literature, these do not readily inform the design of a functional implantable device. In this study, we demonstrated the feasibility of using mathematical models to geometrically characterise soft tissue space that can guide the mechanical construct of the implant. The model estimations match previously recorded soft tissue volumes and are transferable to other patients.

\section{DECLARATIONS}

\section{Authors' contributions}

Conceptualized the study, collected data, drafted the initial and final manuscript, prepared figures: Hasmat S

Collected data, performed image processing, prepared figures, reviewed and revised the manuscript and approved the final manuscript as submitted: Wanasinghe DL

Collected data, performed image processing, prepared figures, reviewed and revised the manuscript and approved the final manuscript as submitted: Cheng $\mathrm{K}$

Reviewed and revised the manuscript and approved the final manuscript as submitted: Suaning GJ

Reviewed and revised the manuscript and approved the final manuscript as submitted: Lovell $\mathrm{NH}$

Reviewed and revised the manuscript and approved the final manuscript as submitted: Low $\mathrm{T}$

Conceptualized the study, reviewed the manuscript and approved the final manuscript as submitted: Clark JR

\section{Availability of data and materials}

The presented manuscript contains the available data for use.

\section{Financial support and sponsorship}

None.

\section{Conflict of interest}

The authors have no conflicts of interest relevant to this article to disclose.

\section{Ethical approval and consent to participate}

Institutional ethics approval was obtained for use of patient data for this study (approval no. HREC LNR18/RPAH/153). All the authors confirm that the presented manuscript conforms to the Declaration of Helsinki.

\section{Consent for publication}

Not applicable.

\section{Copyright}

(c) The Author(s) 2021. 


\section{REFERENCES}

1. Jowett N, Kearney RE, Knox CJ, Hadlock TA. Toward the bionic face: a novel neuroprosthetic device paradigm for facial reanimation consisting of neural blockade and functional electrical stimulation. Plast Reconstr Surg 2019;143:62e-76e.

2. Tollefson TT, Senders CW. Restoration of eyelid closure in facial paralysis using artificial muscle: preliminary cadaveric analysis. Laryngoscope 2007;117:1907-11.

3. Hasmat S, Lovell NH, Suaning GJ, Low TH, Clark J. Restoration of eye closure in facial paralysis using implantable electromagnetic actuator. J Plast Reconstr Aesthet Surg 2016;69:1521-5.

4. Foirest C, Granger B, Gatignol P, et al. Smile reanimation after unilateral facial palsy by lengthening temporalis myoplasty: objective and subjective evaluation on 25 cases. Plast Reconstr Surg 2017;139:984e-93e.

5. Stephan CN, Devine M. The superficial temporal fat pad and its ramifications for temporalis muscle construction in facial approximation. Forensic Sci Int 2009;191:70-9.

6. Zhao Y, Chundury RV, Perry JD. Comparison of temporal fossa volume in young versus senescent human skulls. Ophthalmic Plast Reconstr Surg 2015;31:482-5.

7. Rinkinen J, Agarwal S, Beauregard J, et al. Morphomic analysis as an aid for preoperative risk stratification in patients undergoing major head and neck cancer surgery. J Surg Res 2015;194:177-84.

8. Ranganathan K, Terjimanian M, Lisiecki J, et al. Temporalis muscle morphomics: the psoas of the craniofacial skeleton. J Surg Res 2014;186:246-52.

9. Furtner J, Genbrugge E, Gorlia T, et al. Temporal muscle thickness is an independent prognostic marker in patients with progressive glioblastoma: translational imaging analysis of the EORTC 26101 trial. Neuro Oncol 2019;21:1587-94.

10. Kim JH, Lee R, Shin CH, Kim HK, Han YS. Temporal augmentation with calvarial onlay graft during pterional craniotomy for prevention of temporal hollowing. Arch Craniofac Surg 2018;19:94-101.

11. Huang RL, Xie Y, Wang W, Tan P, Li Q. Long-term outcomes of temporal hollowing augmentation by targeted volume restoration of fat compartments in chinese adults. JAMA Facial Plast Surg 2018;20:387-93.

12. Cervelli D, Gasparini G, Grussu F, et al. Autologous fat transplantation for the temporalis muscle flap donor site: our experience with 45 cases. Head Neck 2014;36:1296-304.

13. Volk GF, Karamyan I, Klingner CM, Reichenbach JR, Guntinas-Lichius O. Quantitative magnetic resonance imaging volumetry of facial muscles in healthy patients with facial palsy. Plast Reconstr Surg Glob Open 2014;2:e173.

14. Senders CW, Tollefson TT, Curtiss S, Wong-Foy A, Prahlad H. Force requirements for artificial muscle to create an eyelid blink with eyelid sling. Arch Facial Plast Surg 2010;12:30-6.

15. Kim S, Matic DB. The anatomy of temporal hollowing: the superficial temporal fat pad. J Craniofac Surg 2005;16:651-4.

16. Furtner J, Berghoff AS, Schöpf V, et al. Temporal muscle thickness is an independent prognostic marker in melanoma patients with newly diagnosed brain metastases. J Neurooncol 2018;140:173-8.

17. Goto TK, Tokumori K, Nakamura Y, et al. Volume changes in human masticatory muscles between jaw closing and opening. $J$ Dent Res 2002;81:428-32.

18. Rezaeitabar Y, Ulusoy I. Automatic 3D segmentation of individual facial muscles using unlabeled prior information. Int J Comput Assist Radiol Surg 2012;7:35-41. 